\title{
Biomechanical Analysis of Walking through a Hallway under Flooded Conditions
}

\author{
Kentaro Kotani $^{1)}$, Yuji Hirato ${ }^{1)}$, Taisuke Ishigaki ${ }^{2)}$, Hiroaki Shimada ${ }^{2)}$, Keiichi Toda ${ }^{3)}$ and Ken Horii ${ }^{1)}$ \\ 1) Faculty of Engineering Science, Kansai University \\ 2) Faculty of Environment and Urban Engineering, Kansai University \\ 3) Disaster Prevention Research Institute, Kyoto University
}

\begin{abstract}
The objective of this study is to measure muscle activity during walking in order to estimate the distance that must be traveled for safe evacuation when the effect of the flow velocity changes. In the present study, electromyogram data were measured for six muscles on the right lower extremity, namely, the rectus femoris, vastus lateralis, long head of the biceps femoris, medial gastrocnemius, tibialis anterior, and gluteus medius. The experimental conditions included walking through a flooded hallway with $0.5-\mathrm{m}$-high water flowing at four different flow velocities $(0,0.47,0.76$, and $1.12 \mathrm{~m} / \mathrm{s}$ in the direction opposite to the walking direction) and walking on dry ground. From the results, the tibialis anterior exhibited the highest muscle activity of up to $85 \%$ MVC with the toe off the ground when the flow velocity was the highest, suggesting that the subject tended to avoid stumbling against water flow during ankle dorsiflexion. The activity of the gluteus medius increased with the flow velocity, suggesting that the subject tried to stabilize the pelvic area to avoid falling. Empirical data for muscle activity during walking under flooded conditions in association with water height and flow velocity will contribute to better understanding safe evacuation from flooded urban underground areas. J Physiol Anthropol 28(1): 23-28, 2009 http://www.jstage.jst.go.jp/browse/jpa2
\end{abstract}

[DOI: $10.2114 / j p a 2.28 .23]$

Keywords: urban flood, evacuation, muscular activity, electromyography, biomechanics, walking posture

\section{Introduction}

Recent floods in urban areas have brought to the fore the importance of evacuation from urban underground areas. Studies have been conducted to estimate the index of evacuation difficulty in underground areas (Ishigaki et al., 2005; Tachi et al., 2001). However, these studies only measured behavioral features such as the time taken to walk the entire length of a simulated walking alley. Our project was conducted in 2 phases: phase 1 involved empirical evaluation of the risk associated with evacuation from flooded underground areas based on biomechanical data, including electromyogram (EMG) data and posture analyses; and phase 2 involved simulation and comparison of the level of risk for the elderly during evacuation under flooded conditions. There are several recent studies investigating lower extremity muscles during walking in water (Barela et al., 2006; Kaneda et al., 2007; Masumoto et al., 2007; Miyoshi et al., 2006). The configuration of the water depth in their studies was, however, relatively greater than what we were interested in; previous water-walking studies set the depth of water at the axilla (Miyoshi et al., 2006) and xiphoid levels (Barela et al., 2006; Masumoto et al., 2007) in order to evaluate the effect of rehabilitation and water calisthenics. However, when evacuating people from urban underground areas, the depth of water is much lesser than those investigated in previous studies since it is not advisable to walk in water during an evacuation operation if the water depth is up to the axilla and xiphoid levels as investigated in the studies for rehabilitation and water calisthenics. The critical depth of water is the level that requires decision making: whether or not the evacuation operation can be performed safely by walking. Currently, the safe evacuation limit is thought to be $50 \mathrm{~cm}$ for an alley (Ishigaki et al., 2004). In addition, we were interested in the influence of the flow velocity on the walking process, since depending on the precipitation rate and the structure of the underground space, the flow velocity may differ in each condition of flooding. In previous research with a water flow mill (Kato et al., 2002; Masumoto et al., 2007), investigators set the flow velocity to approximately $0.8 \mathrm{~m} / \mathrm{s}$, which is lower than the flow velocity under evacuation conditions, where more than $1 \mathrm{~m} / \mathrm{s}$ of water flow may readily be generated. For this purpose, we constructed a hallway equipped with a water pump that could generate various water flow velocities with a water depth of $0.5 \mathrm{~m}$. This paper focuses on the initial phase of our study, wherein we measured the muscle activity during walking in order to estimate the feasibility of a safe evacuation 
operation based on the water height and flow velocity. Further, the relationship between the muscular activity during walking under flooded conditions and the parameters used for the analysis of walking, including stride cycle and the length of stride, was interpreted by means of principle component analysis (PCA).

\section{Methods}

\section{Subjects}

The participants in this study were eight male subjects (mean age: $22.8 \pm 1.0 \mathrm{yr}$ ). Their average height and body mass were $172.9 \pm 5.7 \mathrm{~cm}$ and $63.6 \pm 4.7 \mathrm{~kg}$, respectively. The recruited subjects were healthy individuals without any known musculoskeletal diagnoses. All the subjects were informed about the procedures and potential risks.

\section{Experimental procedure}

A hallway equipped with a water pump that generated water flow was constructed. The experimental session involved walking through a flooded hallway. Figure 1 illustrates the hallway used in the experiment.

Each subject completed all the trials within a day. Throughout the experiment, the average water temperature in the hallway was $16.7^{\circ} \mathrm{C}$ and the average air temperature was $16.6^{\circ} \mathrm{C}$. The experimental conditions included walking in $0.5-$ $\mathrm{m}$-deep water flowing at four different flow velocities $(0,0.47$, 0.76 , and $1.12 \mathrm{~m} / \mathrm{s}$ in the direction opposite to the walking direction, see Fig. 1(a)) and walking on dry ground. Test area 1 was used to analyze flow-velocity conditions of $0 \mathrm{~m} / \mathrm{s}$ and 0.47 $\mathrm{m} / \mathrm{s}$; test area $2,0.76 \mathrm{~m} / \mathrm{s}$; and test area $3,1.12 \mathrm{~m} / \mathrm{s}$ (see Fig. (b) for an overhead view). The $0.5-\mathrm{m}$ water depth was selected because a previous empirical study (Ishigaki et al., 2004) showed that subjects started experiencing difficulties in walking when the water depth was $0.5 \mathrm{~m}$. The flow velocity ranged from 0 to $1.12 \mathrm{~m} / \mathrm{s}$, which covered the range of feasible

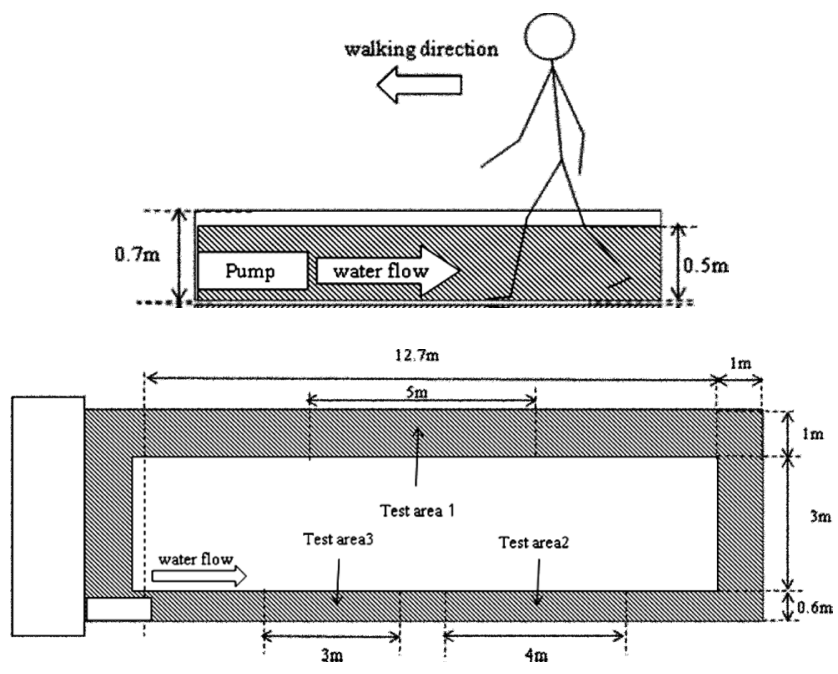

Fig. 1 Test area simulating a hallway. Side view (a) and overhead view (b) of the hallway and the water pump. safe evacuation parameters, as determined by Suga et al. (1995). The experimental order was land walking followed by water walking at flow velocities of $0,0.47,0.76$, and $1.12 \mathrm{~m} / \mathrm{s}$. A total of 5 trials were performed for each condition. No specific walking speed was indicated. Instead, the subjects were instructed to walk at their natural pace. The subjects wore shorts throughout the experiments.

\section{Data analysis}

EMGs were collected for each muscle by using active electrodes (NM-512, Nihon Koden). The electrodes were covered with a waterproof film (BFR10, Nichiban) and waterproof adhesive sealer to provide extensive protection from water damage. The locations for the electrode attachment were prepared by shaving and abrading the skin by using a skin preparation gel, and cleaning the skin with alcohol in order to obtain low inter-electrode impedance. EMG signals were measured for the following six muscles at the right lower extremity: the rectus femoris (RF), vastus lateralis (VL), long head of the biceps femoris (BF), tibialis anterior (TA), gastrocnemius medialis (GM), and gluteus medius (GME). The electrodes were placed on top of the muscle bellies in accordance with Perotto (1994). A reference electrode was attached to the lateral epicondyle of the humerus.

EMG signals were collected using an eight-channel multitelemeter system (WEB-5000, Nihon Koden) and were transferred to a $\mathrm{PC}$ with an A/D converter, which was operated by a bio-signal monitoring system (QP-110H, Nihon Koden). Raw EMG signals were recorded at a sampling rate of $2000 \mathrm{~Hz}$ and filtered with $600 \mathrm{~Hz}$ low- and high-pass filters at a $0.03-\mathrm{s}$ time constant.

The maximum voluntary contraction (MVC) was used to normalize the EMG value across subjects. MVC measurement was conducted in accordance with a regimen introduced by Sawai et al. (2004). The total EMG activity for each muscle was expressed in terms of \%MVC. The subjects performed two sets of MVC trials for each tested muscle, and the higher activity value obtained in the two trials was used as the MVC EMG value. The duration of each MVC trial was $3 \mathrm{~s}$. The subjects rested for $1 \mathrm{~min}$ between trials. MVC data were obtained before the subjects entered the flooded hallway.

The EMG signals were then converted to root-mean-square (RMS) signals, aligned with a stride cycle, and normalized by MVC. The stride cycle was determined by the signal generated using foot switches located under the heel and toe of the right leg. The technique of representing the mean EMG waveform for each experimental condition and muscle location was based on studies dealing with EMG analyses of walking (Kato et al., 2002; Layne et al., 1997; Yang and Winter, 1984). Each EMG waveform was divided into $4 \%$ epochs by representing the mean data within an epoch as a single point; one stride cycle comprised a 25-point ensemble EMG, which was extracted from each trial such that the subjects passed through the center of the test area for the EMG analysis.

Differences in EMG activity were analyzed using a repeated 
measures ANOVA in MINITAB statistical software (Release 14, Minitab Inc.). Significance was noted for probability of a false positive being less than $1 \%$ (i.e., alpha=0.01). The independent variables were walking condition (Land, $0 \mathrm{~m} / \mathrm{s}$, $0.47 \mathrm{~m} / \mathrm{s}, 0.76 \mathrm{~m} / \mathrm{s}, 1.12 \mathrm{~m} / \mathrm{s}$ ), walking phase (stance, swing) and muscle (RF, VL, BF, GM, TA, GME). Subject was included in the model as well.

The PCA was applied to EMG and the performance data in order to reveal the systematic patterns of difficulty in evacuation, which may be indicated in the data. PCA was used for evaluating the relationship among muscular activity and other variables including walking condition, stride cycle, walking speed, stride length, height, and weight. A correlation
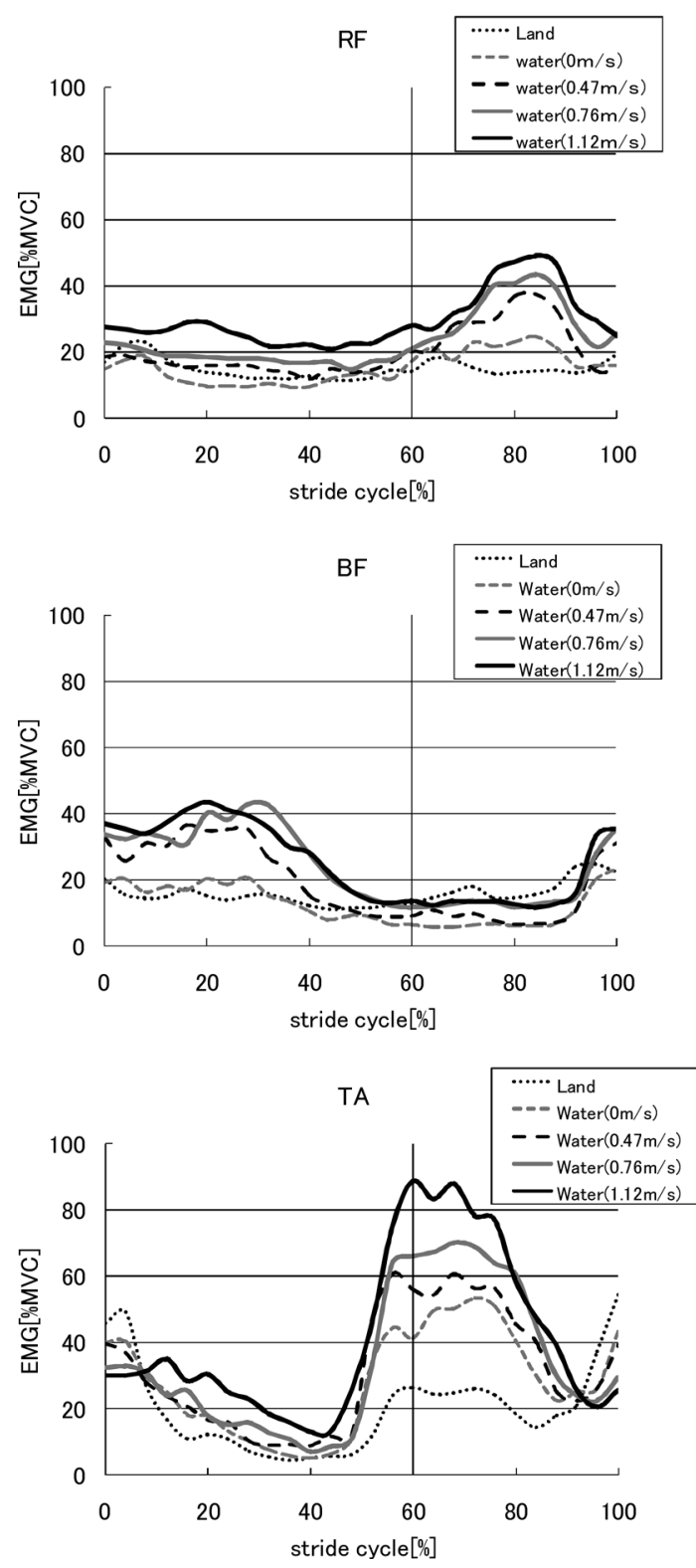

matrix was used for the derivation of the PCA, and factor loadings were obtained from the results of the PCA.

\section{Results}

Figure 2 shows the normalized EMG activities of the six muscles during walking under dry and flooded conditions. The time of walking ranged from 4 to $8 \mathrm{~s}$ approximately. The relative difference in the time of walking was normalized in the case of each subject by their stride cycle. The EMG data were averaged across all subjects. Both the VL (top right in Fig. 2) and RF (top left in Fig. 2), muscles consisting of quadriceps femoris, exhibited a higher activity during the
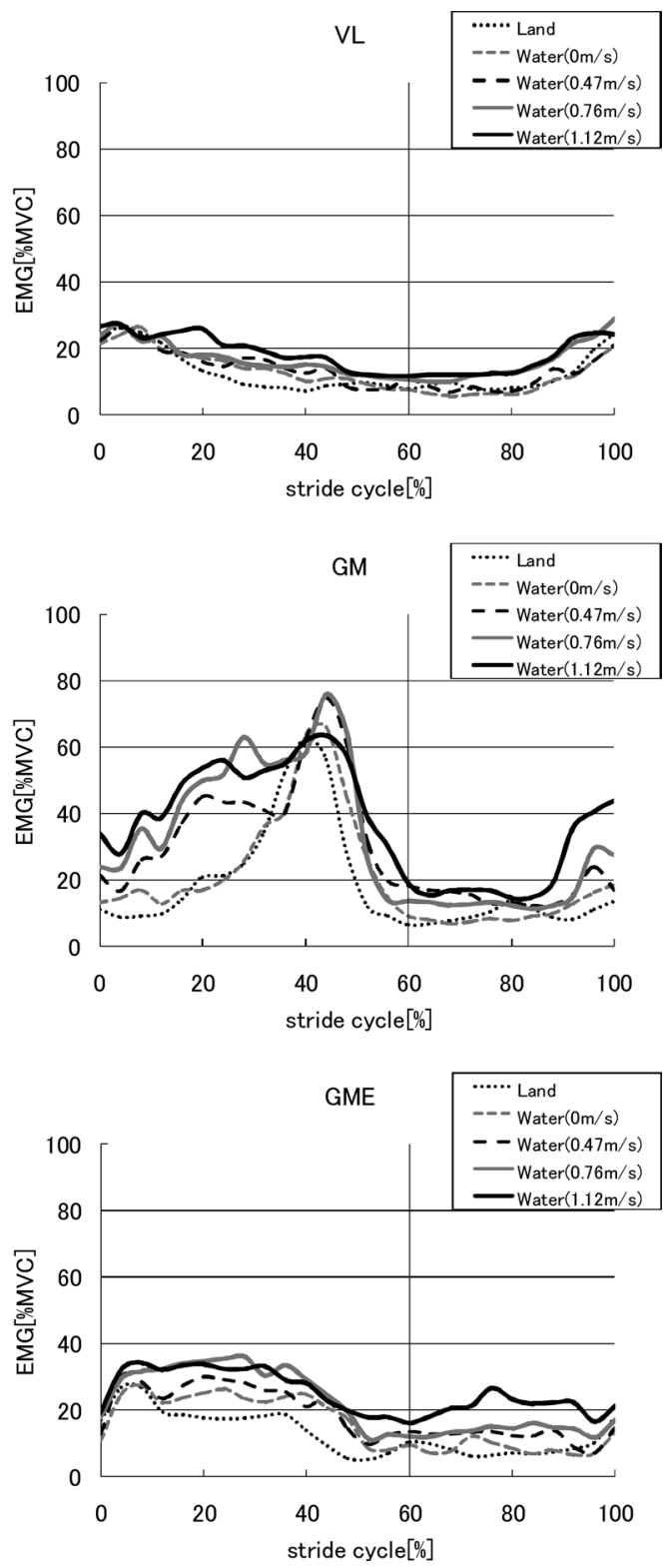

Fig. 2 Muscle activities during walking under different flow velocities (rectus femoris (RF): top left, long head of the biceps femoris (BF): middle left, tibialis anterior (TA): bottom left, vastus lateralis (VL): top right, gastrocnemius medialis (GM): middle right, gluteus medius (GME): bottom right). Vertical lines in each figure indicate separation between the stance phase $(0 \%-60 \%$ of the stride cycle) and the swing phase $(60 \%-100 \%$ of the stride cycle) at $60 \%$ of the stride cycle. 
initial part of the stance phase of walking on dry ground. During walking under flooded conditions, these two muscles demonstrated different tendencies. RF activity increased with the water velocity in the middle of the swing phase. In comparison, VL activity was relatively low, and no difference was observed in VL activity between walking on dry ground and under flooded conditions.

BF (middle left in Fig. 2) activity and the time taken to complete $0 \%-40 \%$ of the stride cycle increased with the flow velocity.

In terms of activity, GM (middle right in Fig. 2) was similar to $\mathrm{BF}$, showing greater activity in longer duration during walking under flooded conditions. Since the GM acts as an ankle plantar flexor, the results indicated that water resistance affects ankle plantar flexion.

TA activity (bottom left in Fig. 2) was affected by the flow velocity in the period between the latter part of the stance phase and the middle part of the swing phase $(50 \%-90 \%$ of the stride cycle).

GME (bottom right in Fig. 2) activity was similar during walking under both flooded conditions and on dry ground. However, the total activity increased with the flow velocity. Since the major function of GME is to stabilize the body for lateral pelvis displacement, an increase in GME activity increased pelvic stability when the flow velocity increased. From the videotaped walking posture, we verified that the upper body swayed more frequently with an increase in the

Table 1 ANOVA results on EMG activity

\begin{tabular}{lrrrrc}
\hline & df & SS & MS & F & $P$ \\
\hline Subject & 7 & 75751 & 10766 & 44.99 & 0.000 \\
Walking & 4 & 53014 & 13254 & 55.39 & 0.000 \\
$\quad$ Condition & & & & & \\
Phase & 1 & 3052 & 3052 & 12.76 & 0.000 \\
Muscle & 5 & 65237 & 13167 & 55.03 & 0.000 \\
Error & 2334 & 558493 & 239 & & \\
Total & 2351 & 755547 & & & \\
\hline
\end{tabular}

flow velocity.

Table 1 shows the results of ANOVA on EMG activity. It revealed that a significant effect of subject was found on all dependent variables, i.e., subject $(\mathrm{F}(7,2334)=44.99, p<0.01)$, muscle $(\mathrm{F}(5,2334)=55.03, p<0.01)$, walking condition $(\mathrm{F}(4$, $2334)=55.39, p<0.01)$, and walking phase $(\mathrm{F}(1,2334)=$ 12.76, $p<0.01$ ).

The principal component loadings obtained by the analysis are shown in Table 1. The average and standard deviation of each parameter are summarized in Table 2. Loadings with absolute values more than 0.5 were selected and are shown in the table. The principal components were extracted on the basis of the criterion that the eigenvalues for the principal components must exceed 1.0. Based on this criterion, four principal components were finally obtained. Their percentage variances were $37.9 \%, 17.9 \%, 15.0 \%$, and $10.6 \%$, respectively. The cumulative percentage variance became $81.3 \%$ of the variation. Compared to the first principal component, the relative contribution for the remaining eigenvalues dropped rapidly.

Individual variations in the case of each muscle were approximately $10 \%$ MVC, but no specific differences in

Table 3 Average and standard deviation of muscular and kinesiological variables

\begin{tabular}{lrr}
\hline \multicolumn{1}{c}{ Parameter } & Mean & SD \\
\hline Walking Constraint & 2.00 & 1.44 \\
Stride Cycle [s] & 1.59 & 0.31 \\
Walking Speed [m/s] & 0.66 & 0.28 \\
BF [\%MVC] & 20.19 & 8.74 \\
GM [\%MVC] & 29.58 & 10.75 \\
GME [\%MVC] & 20.33 & 9.35 \\
RF [\%MVC] & 22.15 & 11.03 \\
Stride Length [m] & 0.49 & 0.11 \\
TA [\%MVC] & 31.82 & 14.10 \\
Height [cm] & 172.88 & 5.48 \\
Weight [kg] & 63.63 & 4.53 \\
VL [\%MVC] & 21.04 & 17.90 \\
\hline
\end{tabular}

Table 2 Principle components loadings of muscular and kinesiological variables for walking under flooded conditions

\begin{tabular}{|c|c|c|c|c|}
\hline & The 1st principal component & The 2nd principal component & The 3rd principal component & The 4th principal component \\
\hline Walking Condition & -0.89 & & & \\
\hline Stride Cycle & -0.73 & & & \\
\hline $\mathrm{BF}$ & -0.58 & & & \\
\hline GM & -0.78 & & & \\
\hline GME & -0.64 & & & 0.53 \\
\hline $\begin{array}{l}\text { Stride Length } \\
\text { TA }\end{array}$ & -0.70 & \multirow{3}{*}{$\begin{array}{r}-0.51 \\
0.70 \\
0.80 \\
\end{array}$} & & \\
\hline \multicolumn{2}{|l|}{$\begin{array}{l}\text { Height } \\
\text { Weight }\end{array}$} & & & \\
\hline Weight & & & & \\
\hline \multicolumn{2}{|l|}{ VL } & & 0.71 & \\
\hline Total variances explained [\%] & ned [\%] & 17.9 & 15.0 & 10.6 \\
\hline
\end{tabular}


activity patterns were observed.

\section{Discussion}

EMG analysis revealed that VL and RF activity showed similar trends in the stride cycle; however, the levels of activity were quite different. RF was more sensitive to the changes in the flow velocity than VL. The difference in the activities of the two muscles could be explained by the association between muscle function and type, that is, VL is a monoarticular muscle that activates the knee extension, whereas RF is a biarticular muscle that activates knee extension and hip joint flexion. It is reasonable to consider that walking on flooded ground requires hip joint extension rather than knee extension, and the results of VL activity therefore suggested that the application of knee extension was relatively low even when the flow velocity increased. These results corresponded, to some extent, with those of Kato et al. (2001). Due to the purpose of rehabilitation, Kato et al. analyzed RF and VL activities during walking in water with a depth around the level of the ensiform cartilage.

BF activity also changed with the flow velocity in a different way. BF increased the activation time, rather than the activity itself, as the flow increased. Masumoto et al. (2004) demonstrated that walking in water, especially when the water level was at waist level, required BF activity as a driving force against the water resistance. Kaneda et al. (2007) also showed that $\mathrm{BF}$ activity was lower during walking on land than that during walking in deep running water, indicating that the range of motion at the knee and hip joints during walking on land was small as compared with that while walking on water. Hence, our results implied that the extension of the hip joint was strongly influenced by the water resistance.

Although the relative changes were small, TA activity seemed to be affected by the flow velocity as well, especially in the latter part of the stance phase and the middle part of the swing phase. Since the phase in which TA activity was high corresponds to ankle dorsiflexion, the result suggested that the subjects took extra care to avoid stumbling when their toe was off the ground. When the flow velocity was $1.12 \mathrm{~m} / \mathrm{s}$, the MVC of TA was more than $80 \%$; this finding led to the hypothesis that the subjects might have fallen due to difficulty in ankle dorsiflexion to shift off the toe or they might have changed their walking strategy. For example, they may have shortened their stride and tried to find rail-like objects for support in order not to fall in an actual flood if the flow velocity exceeded the range we tested.

The TA and GM activities appeared to impact the risk of falling in the elderly. Wolfson et al. (1985) and Whipple et al. (1987) demonstrated that the weakening of the plantar flexion and dorsiflexion forces of the ankle joint was a major cause of falls in the elderly. Masumoto et al. (2007) also found that older subjects showed decreased ankle plantar flexion activity while walking in water. In comparison to the activities measured for other muscles, a higher muscle activity was observed for TA and GM, which operate as ankle dorsiflexor and planter flexor, respectively. The results implied that the elderly face a higher risk of falling as compared to young people and that they might change their walking posture to avoid falling while walking under flooded conditions.

The proportion of variation associated with the first principal component was $37.9 \%$. The components included walking condition, stride length, stride cycle, walking speed, and five muscle locations except VL. This was interpreted as the factor influenced by the changes in the flow velocity because the tendencies for increasing the stride cycle and muscular activity, especially that of TA and GM, and for decreasing the stride length and the walking speed were observed in the principal components.

The proportion of variation for the second principal component was $17.9 \%$. The height, weight, and TA activity were chosen for the component items. The interpretation for this principal component was difficult since the factor loadings describing that TA activity decreased when a subject's height and weight were large. This component could also be interpreted as the height increasing with the weight because the contributions of factor loadings for the height and weight to the principal component were much larger than those for TA activity.

The proportion of variation for the third principal component was $15.0 \%$. The components included the stride length and the muscular activity on VL. VL acts to reduce the impact generated by heel contact (Winter, 1987); therefore, the component seemed to explain the factor for reducing the impact with an increase in the stride length. The activity by RF, which acts as a knee extensor, was not an item for the second principal component, probably because RF acted mostly as a hip flexor than a knee extensor while the subjects were walking in water.

The fourth principal component exhibited $10.6 \%$ of the proportion of variation. This component included items such as RF and GME. GME is known as a stabilizer for the pelvis during walking (Nakamura et al., 2002). RF may have acted as a stabilizer for whole body balance by working as a hip flexor and knee extensor. Ono and Ryushi (2001) found that the elderly required hip flexion/extension and knee extension for dynamic balancing controls. Compared to their study, relatively younger subjects participated in our study. Hence, young subjects can walk under flooded conditions and for this they use RF frequently to resist the water pressure. Thus, the fourth component can be interpreted as whole body balancing.

In conclusion, muscle activities in lower extremities during walking through a flooded hallway revealed the following results. First, TA was the muscle most influenced by the flow velocity. In this study, the subjects experienced difficulty in performing foot dorsiflexion when the flow velocity was 1.12 $\mathrm{m} / \mathrm{s}$. This induced the risk of tripping when their toes were off the ground. Second, walking in flooded conditions particularly required the use of muscles in the foot joints. Determination of factors affecting evacuation difficulty by applying principal 
component analysis revealed the following relationships. GME and RF were the muscles used to balance the body while walking in water. The stride length had a close relationship with VL activity. A complete evaluation of walking under flooded conditions during evacuation from an underground area will be conducted with further study of muscle activity during stair climbing under flooded conditions, and the relationship between muscular activity during walking and factors including the range of joint motion and the hydrodynamic force generated due to resistance while walking under flooded conditions will be examined. There is a limitation in this study: the experimental design is such that only young subjects were tested, for safety reasons. However, a broader age range should be examined, including the elderly, in order to construct guidelines for safe evacuation from flooded hallways in underground areas.

Acknowledgement This study was partially supported by a DPRI Grant for Exploratory Research, 2007 (19H-2), Kyoto University, the Kansai University Grant-in-Aid for progress of research in graduate courses, 2007 and by JSPS.KAKENHI (20310096).

\section{References}

Barela AMF, Stolf SF, Duarte M (2006) Biomechanical characteristics of adults walking in shallow water and on land. J Electromyogr Kinesiol 16: 250-256

Ishigaki T, Nakagawa H, Baba Y (2004) Hydraulic model tests of urban flood considering underground space by using large city model of Kyoto. Annuals of Disas Prev Res Inst 47B: 527-544 [In Japanese with English Abstract]

Ishigaki T, Toda K, Baba Y, Inoue K, Nakagawa H, Yoshida Y, Tagawa H (2005) Experimental study on evacuation from underground space by using real size models. Annuals of Disas Prev Res Inst 48B: 639-646 [In Japanese with English Abstract]

Kaneda K, Wakabayashi H, Sato D, Nomura T (2007) Lower extremity muscle activity during different types and speeds of underwater movement. J Physiol Anthropol 26: 197-200

Kato T, Sugajima Y, Koeda M, Fukuzawa S, Kitagawa K (2002) Electromyogram activity of leg muscles during different types of underwater walking. Adv Exerc Sports Physiol 8: 39-44

Kato T, Sugajima Y, Yamamoto H (2001) Electromyogram activity of leg muscles during underwater walking. Research Reports of Suzuka University of Medical Science 8: 58-68 [In Japanese with English Abstract]

Layne CS, McDonald PV, Bloomberg JJ (1997) Neuromuscular activation patterns during treadmill walking after space flight. Exp Brain Res 113: 104-116

Masumoto K, Takasugi S, Hotta N, Fujishima K, Iwamoto Y (2004) Electromyographic analysis of walking in water in healthy humans. J Physiol Anthropol Appl Human Sci 23: 119-127

Masumoto K, Shono T, Takasugi S, Hotta N, Fujishima K, Iwamoto Y (2007) Age-related differences in muscle activity, stride frequency and heart rate response during walking in water. J Electromyogr Kinesiol 17: 596-604

Miyoshi T, Nakazawa K, Tanizaki M, Sato T, Akai M (2006) Altered activation pattern in synergistic ankle plantarflexor muscles in a reduced-gravity environment. Gait Posture 24: 94-99

Nakamura R, Saito H, Nagasaki H (2002) Fundamental kinesiology. Ishiyaku Publishers, Inc. [In Japanese]

Ono A, Ryushi T (2001) Effects of muscle thickness and strength in the lower limbs on dynamic postural stability in older adults. Jpn J Physiol Anthrop 6: 17-22 [In Japanese with English Abstract]

Perotto AO (1994) Anatomical Guide for the Electromyographer. 3rd ed., Charles C Thomas

Sawai S, Sanematsu H, Kanehisa H, Tsunoda N, Fukunaga T (2004) Evaluation of muscular activity level in daily actions. Jpn Soc Phys Fitness Sports Med 53: 93-105 [In Japanese with English Abstract]

Suga K, Uesaka T, Yoshida T, Hamaguchi K, Chen Z (1995) Preliminary study on feasible safe evacuation in flood disaster. Annual Journal of Hydraulic Engineering 39: 879-882 [In Japanese with English Abstract]

Tachi K, Taketomi H, Mizukusa K, Suetsugi T, Yoshitani J (2001) An experimental study on inundation flow over stairs into underground space. Civil Engineering Journal 43: 22-27 [In Japanese with English Abstract]

Whipple RH, Wolfson LI, Amerman PM (1987) The relationship of knee and ankle weakness to falls in nursing home residents: An isokinetic study. J Am Geriatr Soc 35: $13-20$

Winter DA (1987) The Biomechanics and Motor Control of Human Walking. University of Waterloo Press

Wolfson LI, Whipple R, Amerman P, Kaplan J, Kleiberg A (1985) Gait and balance in the elderly. Two functional capacities that link sensory and motor ability to falls. Clin Geriatr Med 1: 649-659

Yang JF, Winter DA (1984) Electromyographic amplitude normalization methods: Improving their sensitivity as diagnostic tools in gait analysis. Arch Phys Med Rehabil 65: 517-521

Received: November 27, 2007

Accepted: November 7, 2008

Correspondence to: Kentaro Kotani, Faculty of Engineering Science, Kansai University, Suita, Osaka 564-8680, Japan

Phone: +81-6-6368-1121

Fax: +81-6-6330-3154

e-mail: kotani@iecs.kansai-u.ac.jp 